\title{
COMPARATIVE EFFECT OF PROGRESSIVE SPEED TRAINING PROGRAM OF TRIBAL AND NON-TRIBAL BOYS
}

\author{
Chandra Sankar Hazari, \\ Shaybal Chandai, \\ Sumanta Kumar Mondal \\ Department of Physical Education and Sport Science, \\ Institute of Education, Visva-Bharati University, \\ Santiniketan, West Bengal, \\ India
}

\begin{abstract}
:
Speed is one of the vital motor abilities that need to start the developmental process at the early ages of the players. The study aims to identify the progression of progressive speed training basis on the duration of training of the Santali tribe and Bengali teen boys. Subjects were Santali tribe and Bengali adolescent schoolboys and their ages ranged between 13 to 15 years selected from Bankura District of West Bengal, India. These two groups were further divided into control and experimental groups and in each of the groups, there were 20 students. Initially, 4 weeks of uniform conditioning trainings were given to all groups before the pretest T1 was conducted. Further, consecutively 3 more post-tests were conducted every 4 weeks after providing progressive speed training. For the comparison, MANOVA, ANOVA, and LSD post hock test were employed and the Mean value was seen in the descriptive part. The result of the study reveals that Nontribal (Bengali) and Tribal (Santali) adolescent schoolboys responded positively with the designed progressive speed training. This progression of tests timing took place progressively over time on the Bengali and Santali boys almost similarly. Though the Santali boys took the upper hand over Bengali boys numerically at the final stage of progression in the timing of the speed test, on the contrary in the first two post-tests, T2 \& T3 progression took place almost in the same fashion. It is concluded that alike progressive speed training is almost equally effective for Santali tribe and Bengali adolescent boys for the development of sprinting ability.
\end{abstract}

Keywords: speed, training, Santali, Bengali, adolescent

${ }^{i}$ Correspondence: email shaybalchanda@yahoo.com 


\section{Introduction}

Speed in training theory defines the capacity of moving a limb or part of the body's lever system or the whole body with the greatest possible velocity (Dick, 2007). Speed is the ability to move all or part of the body as quickly as possible (BBC, 2021). In this $21^{\text {st }}$ century of the advanced technological era, Coaches need to take cutting edge preparation to bring their athletes at forefront of others. The primary need of all sports is to develop all the motor abilities of the players including speed for maximum output of their sports performance. To keep own athletes edge over the competition, improving their sprinting ability has immense importance (Behrens \& Simonson, 2011). The higher velocity of a sprint only can be achieved by multiplying stride frequency with stride length (Paradisis \& Cooke, 2006). For the development of sprinting speed, both aspects must be improved (Dintiman\& Ward, 2003). Additionally, reaching the utmost velocity as fast as possible is another essential ability of the player that also needs to address appropriately (Zafeiridis et al., 2005). Stride length in sprinting is a mechanical issue of Biomechanics that depends greatly on skeletal and postural structure and economic use of these (Bezodis et al., 2019). The myocellular foundation of the muscles is a determinant factor for success in highlevel sprint competition (Trappe et al., 2015).

Each population groups vary according to their body structure, mass, growth, and figure (Rizvi et al., 2013). These differences exist in different ethnic groups due to the genetic and environmental mysterious amalgamation (Harvey et al., 2010). The major three distinguishable races are Mongoloid or Asiatic, Caucasian or White, and Negroid or Black (Lee, 1943). These groups stand alone based on their different traits. Differences within a racial group do not limit themselves (Bacon et al., 1983; Drummond, 1968; Guo, 1971; Miura et al., 1965) but also distinct features of anthropology and morphology are evidenced in its subdivisions (Holdaway, 1983). Designing a uniform standard for all racial groups and their subdivisions is an unrealistic approach (Kowalski et al., 1974).

Santali population anthropologically belongs to the Proto-Australoid group (Saha et al., 1992). The Santali population is short in height, skin color is dark brown to nearly black, the head is dolichocephalic, the nose is broad \& flat and depressed at the root, hair is wavy and/or curly, and supraorbital ridges are prominent (Malhotra \&Vasulu, 2019). Bengali people originated from an Indo-Aryan ethnic group (Chattopadhyay, 2000). Some other findings also suggest that genetic traces exist in the Bengali population of Proto-Autraloids, Mongoloids, and Caucasoids, these influences shaped Bengali as broad head, dark in complexion, plentiful air on the face, built with medium stature, medium nose (Mukherjee et al., 1987). Therefore, it is clear that there is a difference in anthropology and morphology between Santali and Bengali populations though they share the same land as habitant. Thus, the purpose of the study is to identify whether there is any difference in response to the progressive speed training between Bengali and Santali schoolboys. The study aims at identifying the progression of progressive speed training basis on the duration of training of the Santali tribe and Bengali adolescent boys. 


\section{Material and Methods}

\subsection{Selection of Subject}

Subjects were selected from the Bankura district of West Bengal, India. Subjects were racially Bengali and Santali. These two groups were further divided into control and experimental groups wherein each group 20 subjects were selected randomly. Their age ranged from 13 to 15, and data were collected in the year 2018.

\subsection{Training Protocol}

After random selection of the subjects of each of the groups, they have been given 4 weeks of conditioning training of general nature, then their performance in 60 miter sprint test was recorded. Further, 4 weeks of progressive speed training have been given on experimental groups only in 3 spells and then after every spell of training same test was administered on the experimental and control groups to collect test scores.

Table 1: Pre Test Conditioning Training for All for 4 Weeks

\begin{tabular}{|c|c|c|c|c|}
\hline & Activity & Time & Repetition & Total Time \\
\hline Warming Up & $\begin{array}{l}\text { Jogging } \\
\text { Medium Pace Run } \\
\text { Free-hand Exercise } \\
\text { Rotational Exercise } \\
\text { Stretching Exercise } \\
\text { Short Sprint(with active rest) }\end{array}$ & $\begin{array}{l}2 \mathrm{~min} . \\
2 \mathrm{~min} . \\
1 \mathrm{~min} . \\
1 \mathrm{~min} . \\
2 \mathrm{~min} .\end{array}$ & & $8 \mathrm{~min}$. \\
\hline Acceleration Races & 30-meter dash (with active rest) & $8 \mathrm{~min}$. & 6 times & $8 \mathrm{~min}$. \\
\hline Strength Activities & $\begin{array}{l}\text { Quadriceps And Calf Muscle Exercise } \\
\text { (with active rest) } \\
\text { (i) One leg hopping } \\
\text { (ii) Two-leg hopping } \\
\text { (iii) Box jump } \\
\text { (iv) Running with high knee action } \\
\text { (v) Forward, backward and sideward } \\
\text { running } \\
\text { Abdominal muscle exercise } \\
\text { (with active rest) } \\
\text { (i) Sit-ups } \\
\text { (ii) Flutter kicks } \\
\text { (iii) Squats } \\
\text { (iv) Bicycle crunches } \\
\text { Burpee exercise (with active rest) } \\
\text { (i) Jumping jack } \\
\text { (ii) Frog jump } \\
\text { (iii) Spot jump } \\
\text { (iv) Hop squat burpee }\end{array}$ & $5 \mathrm{~min}$. & & $(5+5+5)=15 \mathrm{~min}$. \\
\hline Agility Run & $\begin{array}{l}\text { (i) Shuttle run (with active rest) } \\
\text { (ii) Potato run (with active rest) }\end{array}$ & $\begin{array}{l}4 \mathrm{~min} . \\
4 \mathrm{~min} .\end{array}$ & $\begin{array}{l}2 \text { times } \\
1 \text { time }\end{array}$ & $8 \mathrm{~min}$. \\
\hline Flexibility Exercises & $\begin{array}{l}\text { (i) All variety stretching exercises } \\
\text { (with active rest) } \\
\text { (ii) stretching exercises with partner } \\
\text { (with active rest) }\end{array}$ & $\begin{array}{l}4 \mathrm{~min} . \\
4 \mathrm{~min} .\end{array}$ & & $8 \mathrm{~min}$. \\
\hline Endurance Run & Continuous run and Interval run & $13 \mathrm{~min}$. & & 13 min. \\
\hline
\end{tabular}




\begin{tabular}{|l|c|}
\hline \hline Grand Total of Time & $60 \mathrm{~min}$. \\
\hline Cool-down & $15 \mathrm{~min}$. \\
\hline Total Session Time & $75 \mathrm{~min}$. \\
\hline
\end{tabular}

Table 2: Progressive Speed Training Schedule

\begin{tabular}{|c|c|c|c|c|c|c|c|c|c|}
\hline \multirow[b]{2}{*}{$\frac{y}{3}$} & \multirow[b]{2}{*}{ 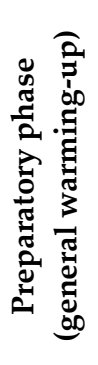 } & \multicolumn{5}{|c|}{ Specific warming up } & \multicolumn{3}{|c|}{$\begin{array}{l}\text { Specific speed } \\
\text { training program }\end{array}$} \\
\hline & & 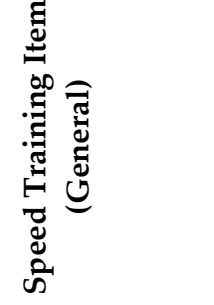 & 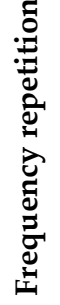 & 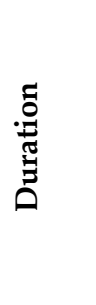 & 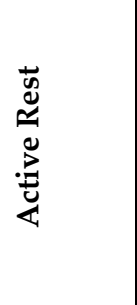 & 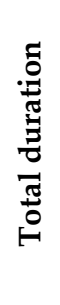 & Day-1 & traming progian & Day-3 \\
\hline 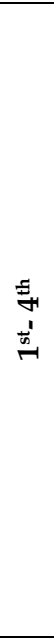 & 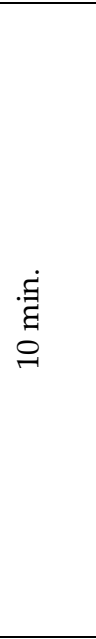 & $\begin{array}{l}\text { 10 Items } \\
\text { Jump squat, } \\
\text { Jump lunges, } \\
\text { Lateral } \\
\text { bound, A } \\
\text { skips, Reverse } \\
\text { lunge knee } \\
\text { drive, Tuck } \\
\text { jump, High } \\
\text { knees, Heel } \\
\text { flicks, } \\
\text { Kneeling } \\
\text { jumps, and } \\
\text { Calf jumps. }\end{array}$ & 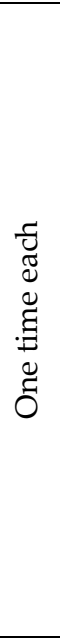 & 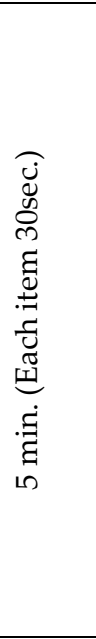 & 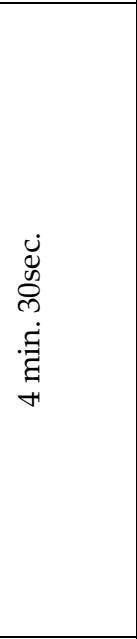 & 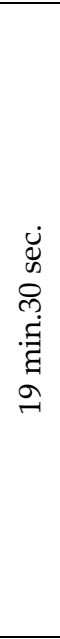 & $\begin{array}{l}\text { 80-meter run, } \\
\text { 90-meter run, } \\
110 \text {-meter } \\
\text { run, } \\
120 \text {-meter } \\
\text { run and 150- } \\
\text { meter run. } \\
\text { (Walk back } \\
\text { recovery } \\
70 \%-80 \% \\
\text { intensity). }\end{array}$ & $\begin{array}{l}\text { ( } 30 \text { meter run } x \\
3 \text { time) x } 2 \\
\text { repetition. } \\
\text { ( } 50 \text { meter run } x \\
2 \text { time) x } 2 \\
\text { repetition. } \\
\text { ( } 80 \text { meter run } x \\
2 \text { time) x } 1 \\
\text { repetition. } \\
\text { (Walk back } \\
\text { recovery } 60 \%- \\
80 \% \text { intensity). }\end{array}$ & $\begin{array}{l}\text { ( } 80 \text { meter run } x \\
3 \text { time) } x 1 \\
\text { repetition. } \\
(90 \text { meter run } x \\
2 \text { time) } \times 1 \\
\text { repetition. } \\
(110 \text { meter run } \\
\text { x } 2 \text { time }) \times 1 \\
\text { repetition. } \\
\text { (120 meter run } \\
\text { x } 1 \text { time) } 1 \\
\text { repetition. } \\
\text { (Walk back } \\
\text { recovery } 60 \%- \\
80 \% \text { intensity). }\end{array}$ \\
\hline $\begin{array}{l}\tilde{\Xi} \\
\infty \\
E^{\prime}\end{array}$ & 离 & Do & 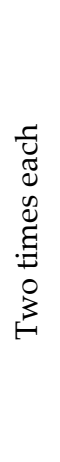 & 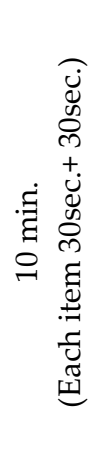 & 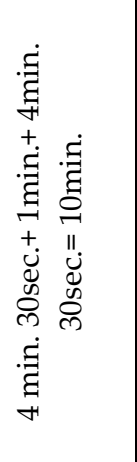 & $\begin{array}{l}\dot{\Xi} \\
\dot{\Xi} \\
0\end{array}$ & $\begin{array}{l}\text { ( } 30 \text { meter run } \\
\text { x } 3 \text { time) } \times 3 \\
\text { repetition. } \\
\text { ( } 40 \text { meter run } \\
\text { x } 2 \text { time) x } 3 \\
\text { repetition. } \\
\text { (50 meter run } \\
\text { x } 1 \text { time) x } 3 \\
\text { repetition. }\end{array}$ & $\begin{array}{l}\text { 110-meter run, } \\
\text { 90-meter run, } \\
\text { 80-meter run, } \\
\text { 90-meter run } \\
\text { and 110-meter } \\
\text { run. } \\
\text { (Sub maximum } \\
\text { effort). }\end{array}$ & $\begin{array}{l}\text { ( } 60 \text { meter run } x \\
2 \text { time) } \times 2 \\
\text { repetition. } \\
(80 \text { meter run } x \\
1 \text { time) } \times 2 \\
\text { repetition } \\
\text { (90 meter run } x \\
2 \text { time) } \times 1 \\
\text { repetition. } \\
\text { (Sub maximum } \\
\text { effort). }\end{array}$ \\
\hline 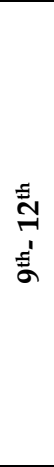 & $\begin{array}{l}\dot{\Xi} \\
\text { ఏ્વ } \\
\stackrel{0}{ }\end{array}$ & Do & 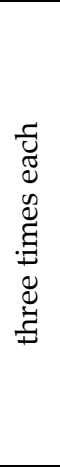 & 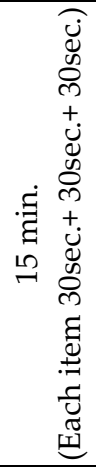 & 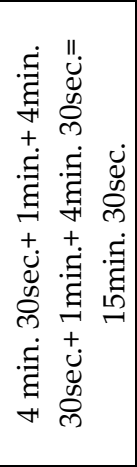 & 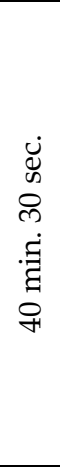 & $\begin{array}{l}\text { (30 meter run } \\
\text { x } 3 \text { time) } \text { x } 4 \\
\text { repetition. } \\
\text { (40 meter run } \\
\text { x } 3 \text { time) x } 4 \\
\text { repetition. } \\
\text { (50 meter run } \\
\text { x } 2 \text { time) x } 2 \\
\text { repetition. } \\
\text { (Full effort). }\end{array}$ & $\begin{array}{l}\text { 80-meter run, } \\
\text { 90-meter run, } \\
\text { 110-meter run } \\
\text { and 120-meter } \\
\text { run. } \\
\text { (Maximum } \\
\text { effort). }\end{array}$ & $\begin{array}{l}\text { (60 meter run } x \\
2 \text { time) } \times \quad 2 \\
\text { repetition. } \\
(80 \text { meter run } x \\
3 \text { time) } \times \quad 1 \\
\text { repetition. } \\
(90 \text { meter run } \times \\
2 \text { time) } \times \quad 1 \\
\text { repetition. } \\
\text { (Maximum } \\
\text { effort). }\end{array}$ \\
\hline
\end{tabular}




\subsection{Materials, Equipment, and Facilities}

For the collection of the timing of 60 miter speed test, standard stopwatch, to record the given performance score sheet, and well-marked track on the grass field were brought in use.

\subsection{Scoring Method}

Timekeepers had been given sufficient enough practice to familiarize themselves with the stopwatch operation well before data collection. Participants took the required individual warm-up time before the 60 meter speed test. They had been given enough verbal instructions and practical demonstrations regarding the test and motivation before taking the test. The timing of a single trial was recorded as a test score.

\subsection{Statistical Procedure}

In the descriptive statistics, Mean valued has been revealed to see numerical differences. To establish the differences in the performances one-way MANOVA \& ANOVA, and LSD as a post hoc test has been employed. The level of significance was $\alpha=0.05$.

\section{Results}

Bar Chart 1: $60 \mathrm{~m}$ Speed Test Mean Timing

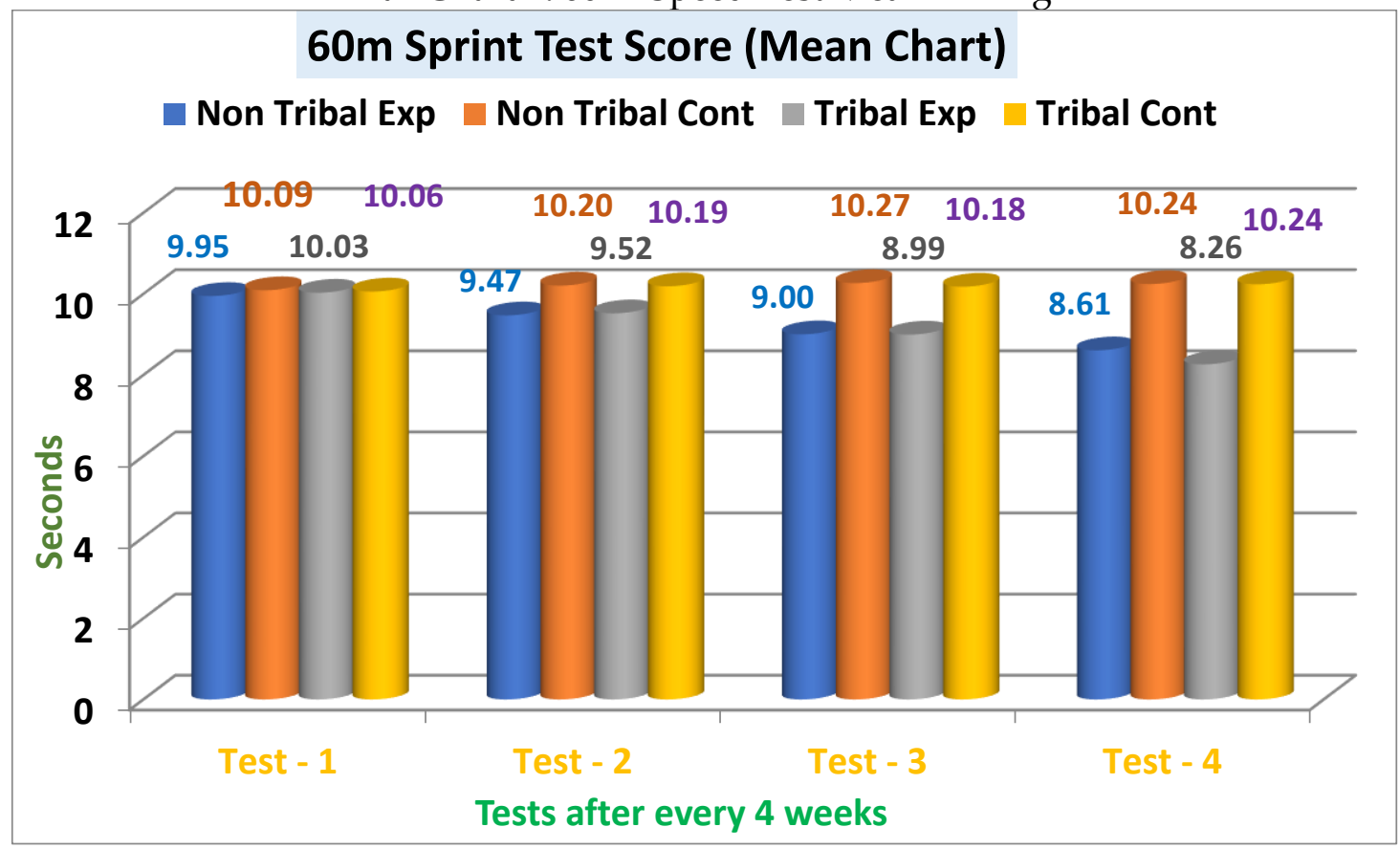

Bar Chart 1 of the mean performances of different four tests of 60-meter Sprint test to measure progressive speed training effectiveness evidenced that both the Control groups of non-tribal $(M=10.09,10.20,10.27,10.24 \mathrm{sec}$. $)$ and Tribal $(M=10.06,10.18,10.18$, $10.24 \mathrm{sec}$.) groups throughout 4 tests performed with a negligible fluctuation in timing. Moreover, between the control groups, all the way did not identify any remarkable 
differences in timing in the test performance. On the other hand, both the experimental groups of Tribal and Non-Tribal progressively improved their mean timing as training progressed whereas, the Tribal group $(M=10.03,9.52,8.99,8.26$ sec. $)$ took upper-hand over the Non-Tribal $(M=9.95,9.47,9.00,8.61 \mathrm{sec}$.) group visibly in the last speed test after 12 weeks of training.

Table 3: MANOVA Test table of $60 \mathrm{~m}$ Speed Test among the Tests

\begin{tabular}{|c|c|c|c|c|c|c|c|c|}
\hline \multicolumn{2}{|l|}{ Effect } & \multirow{2}{*}{$\begin{array}{c}\text { Value } \\
.994\end{array}$} & \multirow{2}{*}{$\begin{array}{c}\mathbf{F} \\
3137.349^{\mathrm{b}} \\
\end{array}$} & \multirow{2}{*}{ 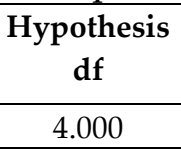 } & \multirow{2}{*}{$\begin{array}{c}\begin{array}{c}\text { Error } \\
\text { df }\end{array} \\
73.000 \\
\end{array}$} & \multirow{2}{*}{$\begin{array}{l}\text { Sig. } \\
.000\end{array}$} & \multirow{2}{*}{$\begin{array}{c}\begin{array}{c}\text { Partial Eta } \\
\text { Squared }\end{array} \\
.994 \\
\end{array}$} & \multirow{2}{*}{$\begin{array}{c}\begin{array}{c}\text { Observed } \\
\text { Power }^{\mathrm{d}}\end{array} \\
1.000 \\
\end{array}$} \\
\hline \multirow{4}{*}{ 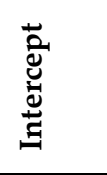 } & Pillai's Trace & & & & & & & \\
\hline & Wilks' Lambda & .006 & $3137.349^{b}$ & 4.000 & 73.000 & .000 & .994 & 1.000 \\
\hline & Hotelling's Trace & 171.910 & $3137.349^{\mathrm{b}}$ & 4.000 & 73.000 & .000 & .994 & 1.000 \\
\hline & Roy's Largest Root & 171.910 & $3137.349^{b}$ & 4.000 & 73.000 & .000 & .994 & 1.000 \\
\hline \multirow{8}{*}{$\begin{array}{l}\mathbf{g} \\
8 \\
8 \\
0 \\
0 \\
0 \\
0 \\
0\end{array}$} & Pillai's Trace & .883 & 7.820 & 12.000 & 225.000 & .000 & .294 & 1.000 \\
\hline & Wilks' Lambda & .166 & 15.685 & 12.000 & 193.431 & .000 & .451 & 1.000 \\
\hline & Hotelling's Trace & 4.746 & 28.343 & 12.000 & 215.000 & .000 & .613 & 1.000 \\
\hline & Roy's Largest Root & 4.684 & $87.823^{c}$ & 4.000 & 75.000 & .000 & .824 & 1.000 \\
\hline & \multicolumn{8}{|c|}{ a. Design: Intercept + Group Sprint $60 \mathrm{~m}$} \\
\hline & \multicolumn{8}{|l|}{ b. Exact statistic } \\
\hline & \multicolumn{8}{|c|}{ c. The statistic is an upper bound on F that yields a lower bound on the significance level. } \\
\hline & \multicolumn{8}{|c|}{ d. Computed using alpha $=.05$} \\
\hline
\end{tabular}

The result of MANOVA Table 2 yielded that there was a statistically significant difference in the four tests together, pretest T1 and pot testes T2, T3, T4 of 60-meter speed training effect after every four weeks of speed training, on the combined dependent variable, Pillai's Trace $=.883, F(12,225)=7.820, p<.001$, partial $\eta^{2}=.294$, observed power $(1-\beta)=$ 1.00. Based on the result, evidence was enough to reject the null hypothesis and conclude that tests combined significantly differed based on the type of the groups participated in. The effect size of the test was large. Cohen (1962, p368) suggests that effect size: small = 0.01, medium $=0.06$, and large $=0.14$ (Schäfer \& Schwarz, 2019; Sullivan \& Feinn, 2012). The observed power was 1.00 , demonstrating that there was a $100 \%$ possibility that the outcome could have come out significant.

Table 4: ANOVA Tests of Between-Subjects Effects

\begin{tabular}{|c|c|c|c|c|c|c|c|}
\hline Source & $\begin{array}{c}\text { Dependent } \\
\text { Variable }\end{array}$ & df & $\begin{array}{l}\text { Mean } \\
\text { Square }\end{array}$ & F & Sig. & $\begin{array}{l}\text { Partial Eta } \\
\text { Squared }\end{array}$ & $\begin{array}{c}\text { Observed } \\
\text { Power }\end{array}$ \\
\hline \multirow{4}{*}{$\begin{array}{l}\text { Group } 60 \mathrm{~m} \\
\text { Sprint }\end{array}$} & Test 1 & 3 & .075 & .107 & .956 & .004 & .069 \\
\hline & Test 2 & 3 & 3.286 & 4.758 & .004 & .158 & .885 \\
\hline & Test 3 & 3 & 9.982 & 15.849 & .000 & .385 & 1.000 \\
\hline & Test 4 & 3 & 22.112 & 38.387 & .000 & .602 & 1.000 \\
\hline \multirow[t]{4}{*}{ Error } & Test 1 & 76 & .698 & & & & \\
\hline & Test 2 & 76 & .691 & & & & \\
\hline & Test 3 & 76 & .630 & & & & \\
\hline & Test 4 & 76 & .576 & & & & \\
\hline
\end{tabular}


Table 4 describes that each ANOVA was tested with Bonferroni method at $\alpha=0.05$ level. Results confirmed that there was enough evidence to reject the null hypothesis for all three post tests, T2: $F(3,76)=4.758, p>0.004$, partial $\eta^{2}=.158$, observed power $(1-\beta)=0.885$; T3: $F(3,76)=15.849, p<.000$, partial $\eta^{2}=.385$, observed power $(1-\beta)=1.000 ; \mathrm{T} 4: F(3,76)=$ 38.387, $p<.000$, partial $\eta^{2}=.602$, observed power $(1-\beta)=1.000$. The effect size was large for all post test ANOVAs. Among the dependent variables' scores of all three post tests T2, T3, \& T4 statistically significant difference exist. In the observed power of $0.885,1.000$, $\& 1.000$ respectively for T2, T3, \& T4 indicated that there were $89 \%, 100 \%$, \& 100\% chances that the result could have come out significant for all analyses.

Table 5: Multiple Comparisons among Dependent Variables within the Test, LSD

\begin{tabular}{|c|c|c|c|c|c|}
\hline $\begin{array}{l}\text { Dependent } \\
\text { Variable }\end{array}$ & $\begin{array}{c}\text { (I) } \\
\text { Group } 60 \mathrm{~m} \\
\text { Sprint } \\
\end{array}$ & $\begin{array}{c}\text { (J) } \\
\text { Group } 60 \mathrm{~m} \\
\text { Sprint } \\
\end{array}$ & $\begin{array}{c}\text { Mean } \\
\text { Difference } \\
(\mathrm{I}-\mathrm{J}) \\
\end{array}$ & $\begin{array}{l}\text { Std. } \\
\text { Error }\end{array}$ & Sig. \\
\hline \multirow{12}{*}{$\stackrel{ت}{\stackrel{1}{\infty}}$} & \multirow{3}{*}{$\begin{array}{l}60 \text { m Sprint } \\
\text { Non-Tribal } \\
\text { Experimental }\end{array}$} & $60 \mathrm{~m}$ Sprint Non-Tribal Control & -.1425 & .26427 & .591 \\
\hline & & $60 \mathrm{~m}$ Sprint Tribal Experimental & -.0805 & .26427 & .761 \\
\hline & & $60 \mathrm{~m}$ Sprint Tribal Control & -.1105 & .26427 & .677 \\
\hline & \multirow{3}{*}{$\begin{array}{l}60 \mathrm{~m} \text { Sprint } \\
\text { Non-Tribal } \\
\text { Control }\end{array}$} & $60 \mathrm{~m}$ Sprint Non-Tribal Experimental & .1425 & .26427 & .591 \\
\hline & & $60 \mathrm{~m}$ Sprint Tribal Experimental & .0620 & .26427 & .815 \\
\hline & & $60 \mathrm{~m}$ Sprint Tribal Control & .0320 & .26427 & .904 \\
\hline & \multirow{3}{*}{$\begin{array}{l}60 \mathrm{~m} \text { Sprint } \\
\text { Tribal } \\
\text { Experimental }\end{array}$} & $60 \mathrm{~m}$ Sprint Non-Tribal Experimental & .0805 & .26427 & .761 \\
\hline & & $60 \mathrm{~m}$ Sprint Non-Tribal Control & -.0620 & .26427 & .815 \\
\hline & & $60 \mathrm{~m}$ Sprint Tribal Control & -.0300 & .26427 & .910 \\
\hline & \multirow{3}{*}{$\begin{array}{l}60 \mathrm{~m} \text { Sprint } \\
\text { Tribal } \\
\text { Control }\end{array}$} & $60 \mathrm{~m}$ Sprint Non-Tribal Experimental & .1105 & .26427 & .677 \\
\hline & & 60 m Sprint Non-Tribal Control & -.0320 & .26427 & .904 \\
\hline & & $60 \mathrm{~m}$ Sprint Tribal Experimental & .0300 & .26427 & .910 \\
\hline \multirow{12}{*}{$\underset{N}{\stackrel{N}{D}}$} & \multirow{3}{*}{$\begin{array}{l}60 \mathrm{~m} \text { Sprint } \\
\text { Non-Tribal } \\
\text { Experimental }\end{array}$} & $60 \mathrm{~m}$ Sprint Non-Tribal Control & $-.7350^{*}$ & .26280 & .007 \\
\hline & & $60 \mathrm{~m}$ Sprint Tribal Experimental & -.0515 & .26280 & .845 \\
\hline & & $60 \mathrm{~m}$ Sprint Tribal Control & $-.7185^{*}$ & .26280 & .008 \\
\hline & \multirow{3}{*}{$\begin{array}{l}60 \mathrm{~m} \text { Sprint } \\
\text { Non-Tribal } \\
\text { Control }\end{array}$} & $60 \mathrm{~m}$ Sprint Non-Tribal Experimental & $.7350^{*}$ & .26280 & .007 \\
\hline & & $60 \mathrm{~m}$ Sprint Tribal Experimental & $.6835^{*}$ & .26280 & .011 \\
\hline & & $60 \mathrm{~m}$ Sprint Tribal Control & .0165 & .26280 & .950 \\
\hline & \multirow{3}{*}{$\begin{array}{l}60 \mathrm{~m} \text { Sprint } \\
\text { Tribal } \\
\text { Experimental }\end{array}$} & 60 m Sprint Non-Tribal Experimental & .0515 & .26280 & .845 \\
\hline & & $60 \mathrm{~m}$ Sprint Non-Tribal Control & $-.6835^{*}$ & .26280 & .011 \\
\hline & & $60 \mathrm{~m}$ Sprint Tribal Control & $-.6670^{*}$ & .26280 & .013 \\
\hline & \multirow{3}{*}{$\begin{array}{l}60 \mathrm{~m} \text { Sprint } \\
\text { Tribal } \\
\text { Control }\end{array}$} & 60 m Sprint Non-Tribal Experimental & $.7185^{*}$ & .26280 & .008 \\
\hline & & 60 m Sprint Non-Tribal Control & -.0165 & .26280 & .950 \\
\hline & & $60 \mathrm{~m}$ Sprint Tribal Experimental & $.6670^{*}$ & .26280 & .013 \\
\hline \multirow{9}{*}{$\stackrel{m}{\infty}$} & \multirow{3}{*}{$\begin{array}{l}60 \mathrm{~m} \text { Sprint } \\
\text { Non-Tribal } \\
\text { Experimental }\end{array}$} & $60 \mathrm{~m}$ Sprint Non-Tribal Control & $-1.2625^{*}$ & .25095 & .000 \\
\hline & & $60 \mathrm{~m}$ Sprint Tribal Experimental & .0075 & .25095 & .976 \\
\hline & & $60 \mathrm{~m}$ Sprint Tribal Control & $-1.1740^{*}$ & .25095 & .000 \\
\hline & \multirow{3}{*}{$\begin{array}{l}60 \mathrm{~m} \text { Sprint } \\
\text { Non-Tribal } \\
\text { Control } \\
\end{array}$} & $60 \mathrm{~m}$ Sprint Non-Tribal Experimental & $1.2625^{*}$ & .25095 & .000 \\
\hline & & $60 \mathrm{~m}$ Sprint Tribal Experimental & $1.2700^{*}$ & .25095 & .000 \\
\hline & & $60 \mathrm{~m}$ Sprint Tribal Control & .0885 & .25095 & .725 \\
\hline & \multirow{3}{*}{$\begin{array}{l}60 \mathrm{~m} \text { Sprint } \\
\text { Tribal } \\
\text { Experimental }\end{array}$} & $60 \mathrm{~m}$ Sprint Non-Tribal Experimental & -.0075 & .25095 & .976 \\
\hline & & $60 \mathrm{~m}$ Sprint Non-Tribal Control & $-1.2700^{*}$ & .25095 & .000 \\
\hline & & $60 \mathrm{~m}$ Sprint Tribal Control & $-1.1815^{*}$ & .25095 & .000 \\
\hline
\end{tabular}


Chandra Sankar Hazari, Shaybal Chanda, Sumanta Kumar Mondal

COMPARATIVE EFFECT OF PROGRESSIVE SPEED TRAINING

PROGRAM OF TRIBAL AND NON-TRIBAL BOYS

\begin{tabular}{|c|c|c|c|c|c|}
\hline & \multirow{3}{*}{$\begin{array}{l}60 \mathrm{~m} \text { Sprint } \\
\text { Tribal } \\
\text { Control }\end{array}$} & $60 \mathrm{~m}$ Sprint Non-Tribal Experimental & $1.1740^{*}$ & .25095 & .000 \\
\hline & & $60 \mathrm{~m}$ Sprint Non-Tribal Control & -.0885 & .25095 & .725 \\
\hline & & $60 \mathrm{~m}$ Sprint Tribal Experimental & $1.1815^{*}$ & .25095 & .000 \\
\hline \multirow{12}{*}{$\stackrel{+}{\stackrel{+}{D}}$} & \multirow{3}{*}{$\begin{array}{l}60 \mathrm{~m} \text { Sprint } \\
\text { Non-Tribal } \\
\text { Experimental }\end{array}$} & $60 \mathrm{~m}$ Sprint Non-Tribal Control & $-1.6350^{*}$ & .24001 & .000 \\
\hline & & $60 \mathrm{~m}$ Sprint Tribal Experimental & .3455 & .24001 & .154 \\
\hline & & $60 \mathrm{~m}$ Sprint Tribal Control & $-1.6290^{*}$ & .24001 & .000 \\
\hline & \multirow{3}{*}{$\begin{array}{l}60 \mathrm{~m} \text { Sprint } \\
\text { Non-Tribal } \\
\text { Control }\end{array}$} & $60 \mathrm{~m}$ Sprint Non-Tribal Experimental & $1.6350^{*}$ & .24001 & .000 \\
\hline & & $60 \mathrm{~m}$ Sprint Tribal Experimental & $1.9805^{*}$ & .24001 & .000 \\
\hline & & $60 \mathrm{~m}$ Sprint Tribal Control & .0060 & .24001 & .980 \\
\hline & \multirow{3}{*}{$\begin{array}{l}60 \mathrm{~m} \text { Sprint } \\
\text { Tribal } \\
\text { Experimental }\end{array}$} & $60 \mathrm{~m}$ Sprint Non-Tribal Experimental & -.3455 & .24001 & .154 \\
\hline & & $60 \mathrm{~m}$ Sprint Non-Tribal Control & $-1.9805^{*}$ & .24001 & .000 \\
\hline & & $60 \mathrm{~m}$ Sprint Tribal Control & $-1.9745^{*}$ & .24001 & .000 \\
\hline & \multirow{3}{*}{$\begin{array}{l}60 \mathrm{~m} \text { Sprint } \\
\text { Tribal } \\
\text { Control }\end{array}$} & $60 \mathrm{~m}$ Sprint Non-Tribal Experimental & $1.6290^{*}$ & .24001 & .000 \\
\hline & & 60 m Sprint Non-Tribal Control & -.0060 & .24001 & .980 \\
\hline & & $60 \mathrm{~m}$ Sprint Tribal Experimental & $1.9745^{*}$ & .24001 & .000 \\
\hline
\end{tabular}

Table 5 explains that each comparison was made with LSD method at $\alpha=.05$ level of significance. LSD comparisons yielded an interesting result that in the post test T2 significant statistical differences have been made in the comparison between Non-Tribal Experimental $(M=9.47)$ with Non-Tribal Control $(M=10.20)$ \& Tribal Control $(M=10.18)$, Non-Tribal Control $(M=10.20)$ \& Tribal Experimental $(M=9.52)$, Tribal Experimental $(M$ =9.52) \& Tribal Control $(M=10.18)$. Further, in the T3 statistically significant differences have been observed in the comparison of Non-Tribal Experimental $(M=9.00)$ with NonTribal Control $(M=10.27)$ \& Tribal Control $(M=10.18)$, Non-Tribal Control $(M=10.27) \&$ Tribal Experimental $(M=8.99)$, Tribal Experimental $(M=8.99)$ \& Tribal Control $(M=$ 10.18). Moreover, in the T4 significant differences have been identified in the comparison of Non-Tribal Experimental $(M=8.61)$ with Non-Tribal Control $(M=10.24)$ \& Tribal Control $(M=10.24)$, Non-Tribal Control $(M=10.24) \&$ Tribal Experimental $(M=8.26)$, Tribal Experimental $(M=8.26) \&$ Tribal Control $(M=10.24)$.

Non-tribal (Bengali) and Tribal (Santali) adolescent schoolboys responded positively to the designed progressive speed training. This progression of timing took place progressively over time on the Bengali and Santali boys almost similarly. Though the Santali boys showed superiority over Bengali boys numerically at the final stage of progression in the timing of the speed test but in the first two post-test T2 \& T3 progression took place almost in the same trend.

\section{Discussion}

Non-tribal (Bengali) and Tribal (Santali) adolescent schoolboys responded positively to the designed progressive speed training. Though there are few anthropological differences exists between these two groups of populations (Malhotra \&Vasulu, 2019; 
Saha et al., 1992) though Proto-Australoid ancestry belongs to populations of both groups (Mukherjee et al., 1987). This progression of timing took place progressively over time on the Bengali and Santali boys almost similarly. Though the Santali boys took the upper hand over Bengali boys numerically at the final stage of progression in the timing of the speed test, on the other hand, in the first two post-tests, T2 \& T3 progression took place almost in a similar pattern. Santali tribe boys might have to take a bit of lead over nontribal Bengali counterparts in the timing of speed test because of comparative more genetic purity (Mukherjee et al., 1987; Saha et al., 1992). Further, Santali students might have an advantage in myocellular structure as it plays the role of determining factor of success in high-level sprinting events (Trappe et al., 2015).

\section{Conclusion}

Alike progressive speed training is almost equally effective for Santali tribe and Bengali adolescent boys for the development of sprinting ability. Coaches may train Santali tribe and Bengali adolescent boys with the identical progressive speed training schedule for the development of sprinting ability.

\section{Conflict of Interest Statement}

The authors declare no conflict of interest.

\section{Source of Funding}

There was no funding body for the study. All expenses related to the study have been borne by the researchers.

\section{Acknowledgment}

All the boys participated in the study as subjects owe indebted thanks from the researchers as they have given a lot of time and effort despite their busy schedule of schooling and homework. Researchers also extend their thanks to the authority of the student concerned school and their guardians to allow subjects to participate in the study.

\section{About the Authors \\ Chandra Sankar Hazari (PhD Scholar), Department of Physical Education and Sport Science, Institute of Education, Visva-Bharati University, Santiniketan, West Bengal, India.}

Shaybal Chanda (PhD Scholar), Department of Physical Education and Sport Science, Institute of Education, Visva-Bharati University, Santiniketan, West Bengal, India.

Sumanta Kumar Mondal (PhD) Professor, Department of Physical Education and Sport Science, Institute of Education, Visva-Bharati University, Santiniketan, West Bengal, India. 


\section{References}

Bacon, W., Girardin, P., \& Turlot, J. C. (1983). A comparison of cephalometric norms for the African Bantu and a Caucasoid population. European Journal of Orthodontics, 5(3), 233-240. https://doi.org/10.1093/ejo/5.3.233

BBC. (2021). The components of fitness - definitions, examples and tests - Keeping fit and healthy in sport-OCR - GCSE Physical Education Revision-OCR. BBC Bitesize. https://www.bbc.co.uk/bitesize/guides/z8j87hv/revision/2

Behrens, M. J. M., \& Simonson, S. R. E. (2011). A Comparison of the Various Methods Used to Enhance Sprint Speed. Strength and Conditioning Journal:,33(2), 64-71. https://doi.org/10.1519/SSC.0b013e318210174d

Bezodis, N. E., Willwacher, S., \&Salo, A. I. T. (2019). The Biomechanics of the Track and Field Sprint Start: A Narrative Review. Sports Medicine, 49(9), 1345-1364. https://doi.org/10.1007/s40279-019-01138-1

Chattopadhyay, A. (2000). A Note on the Contributions of the "Dravidas" to the Ethnicity of the People and Culture of Bengal. Proceedings of the Indian History Congress, 61, 57-64. https://www.jstor.org/stable/44148079

Dick, F. W. (2007). Sports Training Principles (5th ed.). Published by A \& C Black (Publishers) Ltd.

Dintiman, G., \& Ward, R. (2003). Sports Speed (3rd ed.). Human Kinetics.

Drummond, R. A. (1968). A determination of cephalometric norms for the Negro race. American Journal of Orthodontics, 54(9), 670-682. https://doi.org/10.1016/00029416(68)90018-3

Guo, M. K. (1971). Cephalometric standards of Steiner analysis established on Chinese children. Taiwan Yi Xue Hui Za Zhi. Journal of the Formosan Medical Association, 70(2), 97-102.

Harvey, N. C., Mahon, P. A., Robinson, S. M., Nisbet, C. E., Javaid, M. K., Crozier, S. R., Inskip, H. M., Godfrey, K. M., Arden, N. K., Dennison, E. M., \& Cooper, C. (2010). Different Indices of Fetal Growth Predict Bone Size and Volumetric Density at 4 Years of Age. Journal of Bone and Mineral Research: The Official Journal of the American Society for Bone and Mineral Research, 25(4), 920-927. https://doi.org/10.1359/jbmr.091022

Holdaway, R. A. (1983). A soft-tissue cephalometric analysis and its use in orthodontic treatment planning. Part I. American Journal of Orthodontics, 84(1), 1-28. https://doi.org/10.1016/0002-9416(83)90144-6

Kowalski, C. J., Nasjleti, C. E., \& Walker, G. F. (1974). Differential diagnosis of adult male black and white populations. The Angle Orthodontist, 44(4), 346-350. https://doi.org/10.1043/0003-3219(1974)044<0346:DDOAMB>2.0.CO;2

Lee, L. J. (1943). Man's Most Dangerous Myth; The Fallacy of Race. By M. F. Ashley Montagu. (New York: Columbia University Press. 1942. Pp. xiii, 216. \$2.25.). American Political Science Review, 37(1), 168-170. https://doi.org/10.2307/1949023 
Malhotra, K. C., \&Vasulu, T. (2019). Development of typological classification and its relationship to microdifferentiation in ethnic India. Journal of Biosciences. https://doi.org/10.1007/s12038-019-9880-8

Miura, F., Inoue, N., \& Suzuki, K. (1965). Cephalometric Standards for Japanese According to the Steiner Analysis. American Journal of Orthodontics, 51, 288-295. https://doi.org/10.1016/0002-9416(65)90103-x

Mukherjee, B. N., Walter, H., Malhotra, K. C., Chakraborty, R., Sauber, P., Banerjee, S., \& Roy, M. (1987). Population genetic study in ten endogamous groups of West Bengal, India. AnthropologischerAnzeiger, 45(3), 239-254. https://www.jstor.org/stable/29539805

Paradisis, G., \& Cooke, C. (2006). The effects of sprint running training on sloping surfaces. J Strength Cond Res 20: 767-777, 2006. J Strength Cond Res, 20. https://doi.org/10.1519/R-16834.1.

Saha, N., Tay, J. S. H., Roy, A. C., Das, M. K., Das, K., Roy, M., Dey, B., Banerjee, S., \& Mukherjee, B. N. (1992). Genetic Study of Five Populations of Bihar, India. 12. http://library.isical.ac.in:8080/jspui/bitstream/10263/6779/1/Genetic\%20Study\%20 of $\% 20$ Five $\% 20$ Populations $\% 20$ of $\% 20$ Bihar $\% 2$ C $\% 20$ India.pdf

Schäfer, T., \& Schwarz, M. A. (2019). The Meaningfulness of Effect Sizes in Psychological Research: Differences Between Sub-Disciplines and the Impact of Potential Biases. Frontiers in Psychology, 0. https://doi.org/10.3389/fpsyg.2019.00813

Sullivan, G. M., \&Feinn, R. (2012). Using Effect Size-Or Why the P Value Is Not Enough. Journal of Graduate Medical Education, 4(3), 279-282. https://doi.org/10.4300/JGMED-12-00156.1

Trappe, S., Luden, N., Minchev, K., Raue, U., Jemiolo, B., \& Trappe, T. A. (2015). Skeletal muscle signature of a champion sprint runner. Journal of Applied Physiology, 118(12), 1460-1466. https://doi.org/10.1152/japplphysiol.00037.2015

Zafeiridis, A., Saraslanidis, P., Manou, V., Ioakimidis, P., Dipla, K., \& Kellis, S. (2005). The effects of resisted sled-pulling sprint training on acceleration and maximum speed performance. The Journal of Sports Medicine and Physical Fitness, 45(3), 284-290. 

be applied to their work. Under the terms of this license, no permission is required from the author(s) or publisher for members of the community to copy, distribute, transmit or adapt the article content, providing a proper, prominent and unambiguous attribution to the authors in a manner that makes clear that the materials are being reused under permission of a Creative Commons License. Views, opinions and conclusions expressed in this research article are views, opinions and conclusions of the author(s). Open Access Publishing Group and European Journal of Physical Education and Sport Science shall not be responsible or answerable for any loss, damage or liability caused in relation to/arising out of conflict of interests, copyright violations and inappropriate or inaccurate use of any kind content related or integrated on the research work. All the published works are meeting the Open Access Publishing requirements and can be freely accessed, shared, modified, distributed and used in educational, commercial and non-commercial purposes under a Creative Commons attribution 4.0 International License (CC BY 4.0). 